\title{
Pseudo-dynamic method for structural analysis of automobile seats
}

\author{
J O Carneiro ${ }^{1 *}$, F J Q de Melo ${ }^{2}$, J T Pereira ${ }^{3}$, and V Teixeira ${ }^{1}$ \\ ${ }^{1}$ Department of Physics, University of Minho, Campus de Azurém, Guimaraes, Portugal \\ ${ }^{2}$ Department of Mechanical Engineering, TEMA Unit of Mechanical Technology and Automation, University of Aveiro, \\ Campus de Santiago, Aveiro, Portugal \\ ${ }^{3}$ INEGI, Institute of Mechanical Engineering and Industrial Management, Porto, Portugal
}

The manuscript was received on 20 October 2004 and was accepted after revision for publication on 25 May 2005.

DOI: $10.1243 / 146441905 X 52964$

\begin{abstract}
This work describes the application of a pseudo-dynamic (PsD) method to the dynamic analysis of passenger seats for the automotive industry. The project of such components involves a structural test considering the action of dynamic forces arising from a crash scenario. The laboratory certification of these automotive components consists essentially on the inspection of the propagation and extension of plastic deformations zones in metallic members of the seat structure as consequence of the mutual action between the seat and the passenger fastened to the seat via seat belt anchorages. This work presents a relatively simple experiment using PsD techniques as a novel method to perform a test equivalent to the dynamic model of a dummy-seat pair subjected to impulsive loads from a car crash.

Essentially, the PsD test method is a hybrid and hierarchic computer-driven testing procedure where a numerical algorithm and experimental step are used and combined on-line in order to solve a problem in the scope of structural dynamics. The implementation of the method is not expensive and has the leading advantage of offering the operator a total control of any intermediate structure state during the test still keeping the realism of a real dynamic testing.
\end{abstract}

Keywords: pseudo-dynamic method, automobile seats, passenger safety

\section{INTRODUCTION}

The area of transport engineering considers passenger safety as a leading goal. To meet this requirement, a high engineering standard concerning the body design is involved. In order to assess accurately the structural integrity of an automobile body or any integrating element, the designers subject such components to dynamic actions, creating the more realistic mechanical situations arising from an accident. The particular case of the structural assessment of automobile seats and safety belt anchorages of a van/minivan vehicle, classified as $M_{2}$ category according to Council Directive 70/156/EEC, has the procedure of certification test defined by Council Directive 76/115/EEC concerned with the dynamic analysis of components.

*Corresponding author: Department of Physics, University of Minho, Campus de Azurém, 4800-058 Guimaraes, Portugal.
The test rig used for the assessment of the dynamic behaviour of the component in study consists of a rigid frame where the test specimen (the passenger seat) is fastened, according to the design specifications. A pair of high strength $R E N O L D^{\circledR}$ chains connected to the hydraulic cylinder rams pull the seat structure at two levels, respectively, the human torso (the diagonal chest seat-belt) and the waist level (the abdominal seat-belt). The traction devices referred here are depicted in Fig. 1.

The application of forces via the traction devices is achieved with a pair of long stroke hydraulic cylinders, as mentioned. Such a system of forces is equivalent to the inertial loads of the dummy masses having a lumped distribution, respectively, at the torso and the waist levels of the test specimen. In a real time dynamic experiment, the external forces prescribed in the test are equivalent to the inertial effects from the lumped masses mentioned under an acceleration related with a car crash. The accurate 

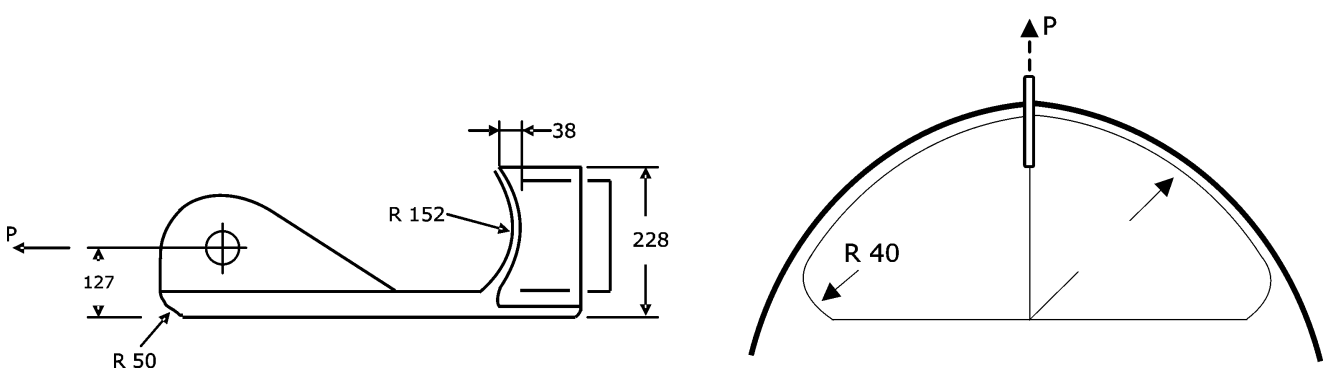

Fig. 1 Traction devices (from Council Directive 76/115/EEC)

modelling of such equivalent loads to prescribe in the test specimen demands that a considerable oil flow should be delivered to the cylinders. This is possible only with hydro-pneumatic accumulators having an adequate oil capacity. The conventional operation mode of this type of experimental set-up consists of an open loop sequence, where once the experiment is started, the operator can only assess the final state of the specimen; there is no control on the test during its intermediate time-steps. The experiment stops when a set of parameters controlling such situation reaches preset values. These may involve a specified time interval for the applied loads or even a substantial decrease in reaction forces level.

\section{PsD TEST METHOD}

\subsection{Numerical algorithm in the PsD method}

The PsD method is a less conventional tool in dynamic analysis operating in a closed loop operation diagram. At each time-step, a structure vector status is recorded at an experimental task and fed back to a computer program running a direct time integration algorithm for the structure dynamic analysis. A new structure configuration is computed in a next time-step, which is prescribed to the structure in test via the test rig actuators. The operating principles of PsD techniques impose some limitations to the type of degrees of freedom (DOF) prescribed to the structure in the test rig. For a convenient performance of this technique, the displacement field should consist mainly in horizontal relative motions between each DOF of the structure in test.

The PsD method is a hybrid process combining the numerical algorithm of the integration of the structure equations of motion [1] and the experimental evaluation of the corresponding structure internal restoring forces. As the applied external and the inertial force system have to be numerically inserted in the procedure, a reduced hydraulic power is required in the test rig; also, the process is performed within a virtual time scale, which, in fact, corresponds to an expansion of the elapsed time referred to the real dynamic test. This means that the equipment used in this procedure runs a test in a quasi-static process rather in a dynamic mode, in spite of dealing with the effective structure dynamic configuration at each time-step. The hydraulic actuators impose a set of prescribed displacements at the level of the structure DOF of the test-specimen, while the load cell set mounted at the cylinder rams record and feed back to the computer algorithm, the structure internal restoring force vector [2-6]. The equation of motion is solved in an on-line process using a stepby-step numerical integration algorithm dealing with the physical forces from the specimen (at the experimental step of the method) and the inertial data from the inertial force vector from the analytical model.

Other contributions have been presented recently, either in the design of test rigs or in the special applications of the technique, other than seismic analysis, such as piping engineering [7] with detail to signal processing equipment and the test rig design to meet the requirements of the geometry of the test specimen and its DOF.

The algorithm broadly used in PsD methods consists essentially in a modified version of the Newmark method. The Hilber et al. [8] formula used in this analysis assumes formerly that the structural damping plays implicitly a joint role with the structure constitutive forces when in the experiment the internal restoring force vector is read from the load cells at the level of the DOF. Usually, as simplification of the procedure, it is assumed that a speed-dependent viscous damping component is neglected. In fact, given the low velocity operation of the displacement actuators, that component is not relevant and non-detectable in the experiment. In contrast, a component due to internal frictions generated during the distortion of the test specimen parts is usually integrated in the concept of structural damping, a less depending component on the speed deformation and possible to be detected during the PsD test. To enhance the contribution of this component, an experiment was performed by the authors of this work, consisting of a harmonic vibration of an 
SDOF structure consisting on a simple built-in beam subject to a forced vibration from a harmonic load [9]. This component is not detected if the structure deformation is carried out in a very slow rate and inserting pauses in order that the internal friction is effectively removed from the experiment. For a multiple degree of freedom (NDOF) system, the algorithm consists in solving iteratively the discrete system of equations

$$
\begin{aligned}
& \mathbf{m} \boldsymbol{a}_{i+1}+\boldsymbol{r}_{i+1}=f_{i+1} \\
& \boldsymbol{v}_{i+1}=v_{i}+(1-\gamma) \Delta t \boldsymbol{a}_{i}+\gamma \Delta t \boldsymbol{a}_{i+1} \\
& \boldsymbol{d}_{i+1}=\boldsymbol{d}_{i}+\Delta t v_{i}+\left(\frac{1}{2}-\beta\right) \Delta t^{2} \boldsymbol{a}_{i}+\beta \Delta t^{2} \boldsymbol{a}_{i+1}
\end{aligned}
$$

where $\mathbf{m}$ is the system mass matrix. The external force vector and internal restoring force vector (this corresponding to product $k \times d$ in case of linearelastic behaviour) of the NDOF system are, respectively, $\boldsymbol{f}_{i+1}$ and $\boldsymbol{r}_{i+1}$ determined at discrete time instants $t_{i+1}=(i+1) \Delta t$, where $\Delta t$ is the time-step interval; the acceleration, velocity, and displacement vectors of the NDOF system are, respectively, $\boldsymbol{a}_{i+1}$, $\boldsymbol{v}_{i+1}$, and $\boldsymbol{d}_{i+1}$ which are also determined at the same time instants. In equation (1), the system mass matrix and external force vector are defined numerically, whereas the internal restoring force vector is experimentally measured from a set of load cells mounted in the rams of the hydraulic actuators. The constants $\gamma$ and $\beta$ included in the generalized Newmark method play their role on selecting an iterative version of the algorithm. For example, if the pair $\beta=0.25$ and $\gamma=0.5$ is adopted, then the Newmark constant acceleration version is achieved [10]. To implement the algorithm in a PsD formulation, it is necessary to reformulate equation (3). Substituting equation (1) into equation (3), the expression for the displacements can be written as

$$
\boldsymbol{d}_{i+1}=\hat{\boldsymbol{d}}_{i+1}-B \boldsymbol{r}_{i+1}
$$

where

$$
\hat{\boldsymbol{d}}_{i+1}=\boldsymbol{d}_{i}+\Delta t v_{i}+\left(\frac{1}{2}-\beta\right) \Delta t^{2} \boldsymbol{a}_{i}+\beta \Delta t^{2} \mathbf{m}^{-1} \boldsymbol{f}_{i+1}
$$

represents the explicit part of the displacement $\boldsymbol{d}_{i+1}$, because all terms in equation. (5) can be calculated with available information from the previous timestep. The constant $B$ is defined as

$$
B=\mathbf{m}^{-1} \beta \Delta t^{2}
$$

Equation (4) represents the implicit form for the displacement, as it can be noticed that the last term on the right-hand side cannot be calculated at time $(i+1) \Delta t$. In fact, as actuators displace the specimen, the restoring forces also change continuously, whereas $\boldsymbol{r}_{i+1}$ is unknown at the beginning of the time-step. This apparent dilemma is overcome on performing the following tasks at each time-step [11].

1. At each iteration, there is a predictor phase and a corrector phase. In the first one, the predictor displacement (i.e. the explicit displacement) $\hat{\boldsymbol{d}}_{i+1}$ is computed from equation (5) and this displacement vector is prescribed to the test specimen via the hydraulic actuators.

2. As soon as the specimen is displaced by the actuators in order to achieve the desired displacements $\boldsymbol{d}_{i+1}$, an intermediate restoring force vector $\boldsymbol{r}$ and displacements $\boldsymbol{x}$ change continuously. Meanwhile, the controller is continuously monitoring a corrective driving parameter, $\boldsymbol{e}=\hat{\boldsymbol{d}}_{i+1}$ $-(x+B r)$; this corresponds to the corrector phase.

3. After the end of the time-step, the corrective parameter should vanish; then when $\boldsymbol{e}=0$, the measured displacement $\boldsymbol{x}=\hat{\boldsymbol{d}}_{i+1}-B \boldsymbol{r}_{i+1}$ matches $\boldsymbol{d}_{i+1}$ corresponding to the desired actual displacement vector.

4. Using equations (1) and (2) for the calculation of actual system acceleration and velocity vectors, the completion of the actual structure kinetic state is achieved; then the data are sent to the actuator control unit and the procedure is repeated from step 1.

Figure 2 shows the set-up for the method, where the operation sequence diagram is represented for an automobile seat test specimen. In the two parallel systems, it is detailed that there is an intercommunication between the step-by-step integration algorithm and the experimental set-up. The first system computes the displacements and sends such information to the second system; the hydraulic actuators (placed at the level of the system DOF) apply the displacements to the test specimen and reply with the restoring forces which is read from the load cells (mounted in each actuator). The first system receives this information and computes the displacements at the next time-step. Although the PsD method follows the same steps as a full-numerical dynamic analysis, it uses the real stiffness and damping characteristics of the specimen.

\subsection{Physical model used in PsD algorithm}

The present analysis used a quite simple structure discretization, which consisted in defining as 'macroelements' the seat basis and the back holder structurally related to the inertial action from chest/ head and hip/legs mass via the diagonal and lower fastening of safety belt, respectively. In order to 


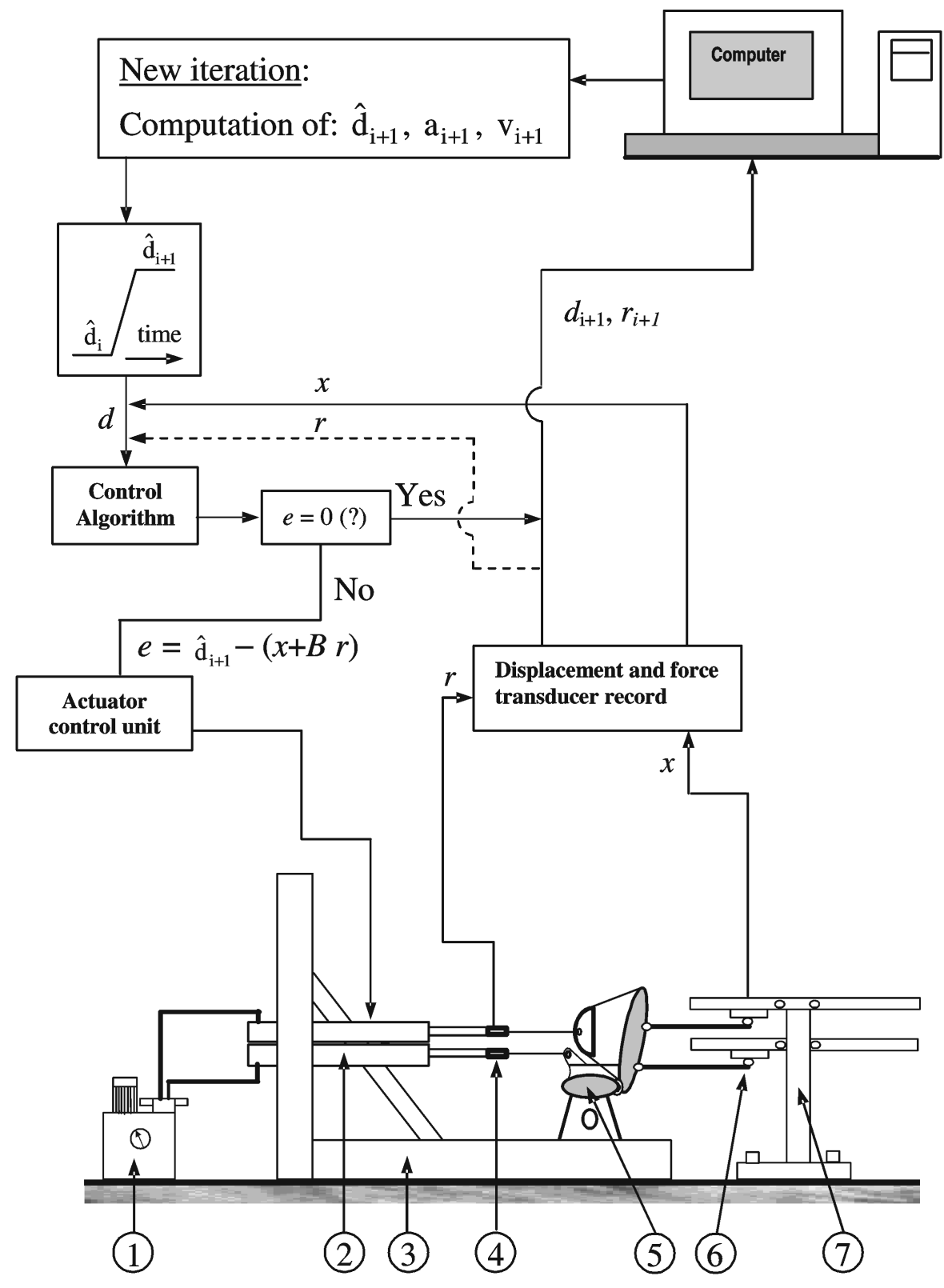

Fig. 2 Diagram of sequences in PsD method: (a) hydraulic power unit, (b) hydraulic cylinder, (c) test rig, (d) load cell, (e) built-in test specimen, (f) linear potentiometer, and (g) reference frame

accurately relate the displacement of the head/chest mass with the corresponding back holder structure (which in fact should be the same) and of hip/legs mass with seat basis, the experiment used a high stiffness steel cable replacing the action of the diagonal strap of safety belt, as shown in Fig. 3.

A simple two-degree-of-freedom model (2DOF) of the specimen was considered to implement the PsD algorithm as seen in Fig. 4. The specimen was modelled as a 2DOF cantilever beam, with an analytically $2 \times 2$ lumped mass matrix formulation associated to the effect of inertial masses coupled with the inertial mass contribution assigned to each DOF of the discretized structure of the seat. It is noted that the inertial forces from the dummy body are here associated with the external force vector prescribed in the Council Directive 70/156/EEC. The elements of the lumped mass matrix were assigned as $\mathbf{M}_{11}=46 \mathrm{~kg}$, $\mathbf{M}_{12}=\mathbf{M}_{21}=0 \mathrm{~kg}$, and $\mathbf{M}_{22}=12 \mathrm{~kg}$, respectively, for DOF ' 1 ' (hip-leg level) and '2' (head-chest level).

The stiffness matrix of this simple structure was experimentally evaluated using a procedure where one of the hydraulic cylinder pair was held in position, whereas the other was driven up to a prescribed displacement. The so-described operation leads to the set-up of a $2 \times 2$ stiffness matrix, where the 

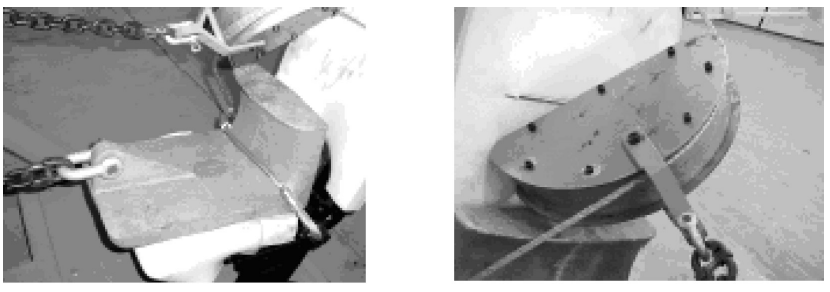

Fig. 3 Body masses and traction devices

leading diagonal elements are the measured forces corresponding to the stiffness associated to each of the seat parts (base or back holder). Such read force values must be normalized, after dividing them by the intensity of the prescribed displacement.

From the assumption of a 2DOF dynamic model for the structure in test, the experimentally evaluated stiffness matrix of the seat test specimen is expressed in SI units $(\mathrm{N} / \mathrm{m})$ as follows:

$$
[\mathbf{k}]=\left[\begin{array}{rr}
156800 & -70560 \\
-70560 & 50960
\end{array}\right]
$$

Naturally, the experimental stiffness matrix evaluation has shown an expected symmetry. It is noted that in equation (7), the element $\mathbf{k}_{11}=156800 \mathrm{~N} / \mathrm{m}$, for example, refers to the necessary force in displacing the DOF 'no. 1' (at the hip/legs or waist level) of $1 \mathrm{~m}$, while keeping DOF no. 2 perfectly still, reacting with a force $\mathbf{k}_{12}=-70560 \mathrm{~N} / \mathrm{m}$; conversely, $\mathbf{k}_{22}=50960 \mathrm{~N} / \mathrm{m}$ refers to the necessary force to displace DOF no. 2 (at the chest/head level) while keeping DOF no. 1 perfectly still and reacting with a force $\mathbf{k}_{12}=\mathbf{k}_{21}=-70560 \mathrm{~N} / \mathrm{m}$. In equation (7), it is noted that $\mathbf{k}_{22}$ assigned to the stiffness of the seat back holder is the more flexible part, about onethird of the lower structure holding the seat to the platform body vehicle, a useful attribute in reducing during a car crash the compression force withstood by the passenger hold in his position by the seat belt.

The test-specimen stiffness matrix is necessary only to select the time-step interval used in the Hilber algorithm. The critical time-step is calculated according to the following criterion

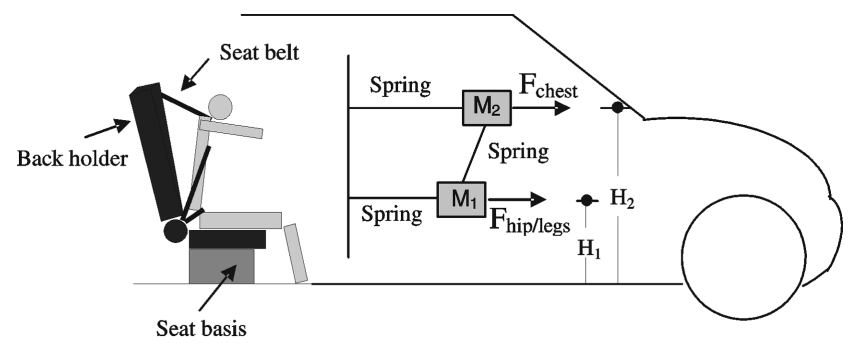

Fig. 4 Passenger car seat and equivalent dynamic model (a) For an SDOF system [12]

$$
\Delta t=\frac{2 \pi}{10} \sqrt{\frac{\mathbf{M}}{\mathbf{K}}}
$$

(b) For an NDOF system, the critical time-step was taken to be $1 / 10$ of the smallest period $T$ associated to the highest natural frequency of the system.

In order to obtain the 2DOF system natural frequencies, the dynamic free vibration equation [13] is solved

$$
\left(\mathbf{k}-\omega^{2} \mathbf{m}\right) \boldsymbol{\psi}=0
$$

The solutions of equation (9) are the natural frequencies of the vibrating system and the associated mode shapes $\psi$ are the eigenvectors. Substituting the lumped mass and stiffness matrices in equation (9), the calculated system natural circular frequencies are, respectively, $\omega_{1}=28.27$ and $\omega_{2}=82.94 \mathrm{rad} / \mathrm{s}$, associated with natural periods $T_{1}=0.22$ and $T_{2}=0.076 \mathrm{~s}$. The critical time-step was then $\Delta t=0.007 \mathrm{~s}$.

\section{PsD TEST SIMULATION OF A TIME-DEPENDENT LOAD ON AN AUTOMOBILE SEAT}

The procedure described in section 2.1 sets the basis of PsD techniques applied to structural dynamics. The technique is recommended to perform a dynamic analysis of simple structures, where its dynamic behaviour can be approached with a low number of DOF. The aim of the PsD experiment carried out in this work is the evaluation of the displacement and internal force response of the automobile seat subjected to a time-dependent load. The external force $\boldsymbol{F}(t)$ is applied at the head/ chest and hip/leg levels along horizontal directions in order to simulate the inertial effects of the passenger against the seat via the seat-belt fasteners. The force system depends on time $t$ as depicted in Fig. 5. It increases linearly for a period of $100 \mathrm{~ms}$, reaching a maximum value of 6.7 and $8.7 \mathrm{kN}$ for the chest and leg levels, respectively. The maximum values are maintained for another $100 \mathrm{~ms}$. The basic time-step interval is $\Delta t=7 \mathrm{~ms}$ as calculated from equation (4).

The experimental set-up used in the work and sketched in Fig. 2 is shown in Fig. 6 where the experimental test rig and some elements are identified, as is the case of hydraulic power unit (right side) and servo hydraulic actuators. 


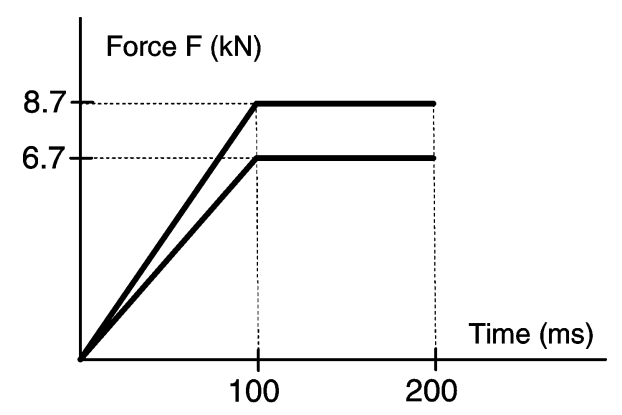

Fig. 5 Time-dependent force acting horizontally at the considered head/chest and hip/leg levels

The designed test rig consists of an L-shaped structure with diagonal stiffeners to improve structure strength. The structure is made of welded steel with a rectangular base $\left(2.84 \times 1.84 \mathrm{~m}^{2}\right)$ and a total height of $2.0 \mathrm{~m}$. The vertical beams have a set of fixtures to receive two servo hydraulic actuators. The active part of the rig consists of double effect hydraulic cylinders (each with a maximum force of $30 \mathrm{kN}$ ) driven by a servo valve. This component is integrated in a hydraulic circuit with other essential elements for the cylinder drive, as is the case of precision gear pump and flow regulators, these preventing spurious cylinder ram displacements between time-steps when under loading. Both hydraulic cylinders can be driven simultaneously; therefore, inducing a nodal displacement field. The displacement transducers have a resolution of $0.001 \mathrm{~mm}$ and a stroke of $\pm 500 \mathrm{~mm}$ for position feedback. An automated system, also forming an integrated unit with the test rig, is coupled to the computer running the program of the Hilber algorithm. The automated system receives at each time-step the displacement data from the computer and prescribes it to the hydraulic actuator, thus defining a next-time step program

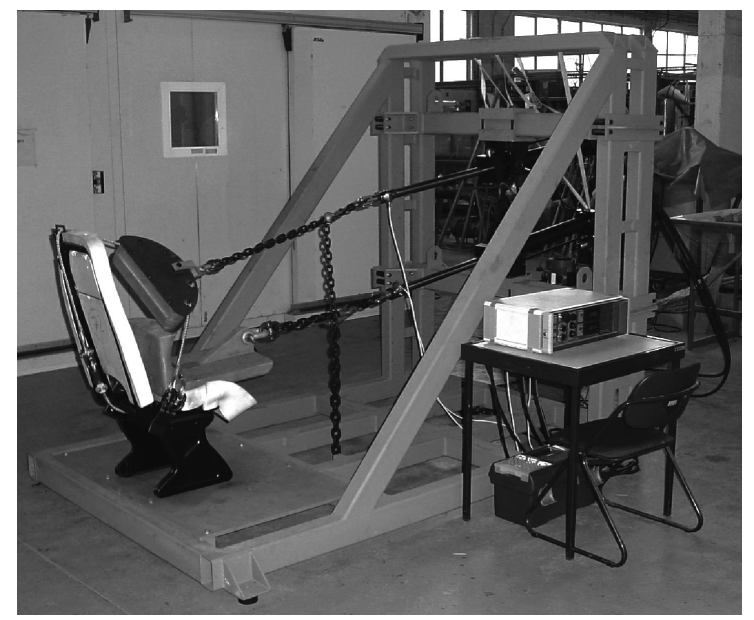

Fig. 6 Layout of the test rig used for PsD test of automotive seats status. The position accuracy of the actuator is ensured by the automated system via a feedback readout received from the linear potentiometer, which is decoupled from the test-rig in order to suppress position errors. In contrast, the automated system sends to the computer the values of the internal restoring forces read from the load cells, as described in section 2.1.

Figures 7(a) and 7(b) show the time evolution of horizontal displacements and internal forces, respectively, corresponding to the two sections of the seat, as a result from the external force, acting as described.

Observing the evolution of internal restoring forces in the structure, it can be detected that yield starts at chest support level past $\cong 80 \mathrm{~ms}$ after the external force action; while the seat basis practically withstood the load increase in the linear elastic zone. The final deformed shape presented a considerable level of distortions, which may be considered a favourable feature for passenger injury reduction.
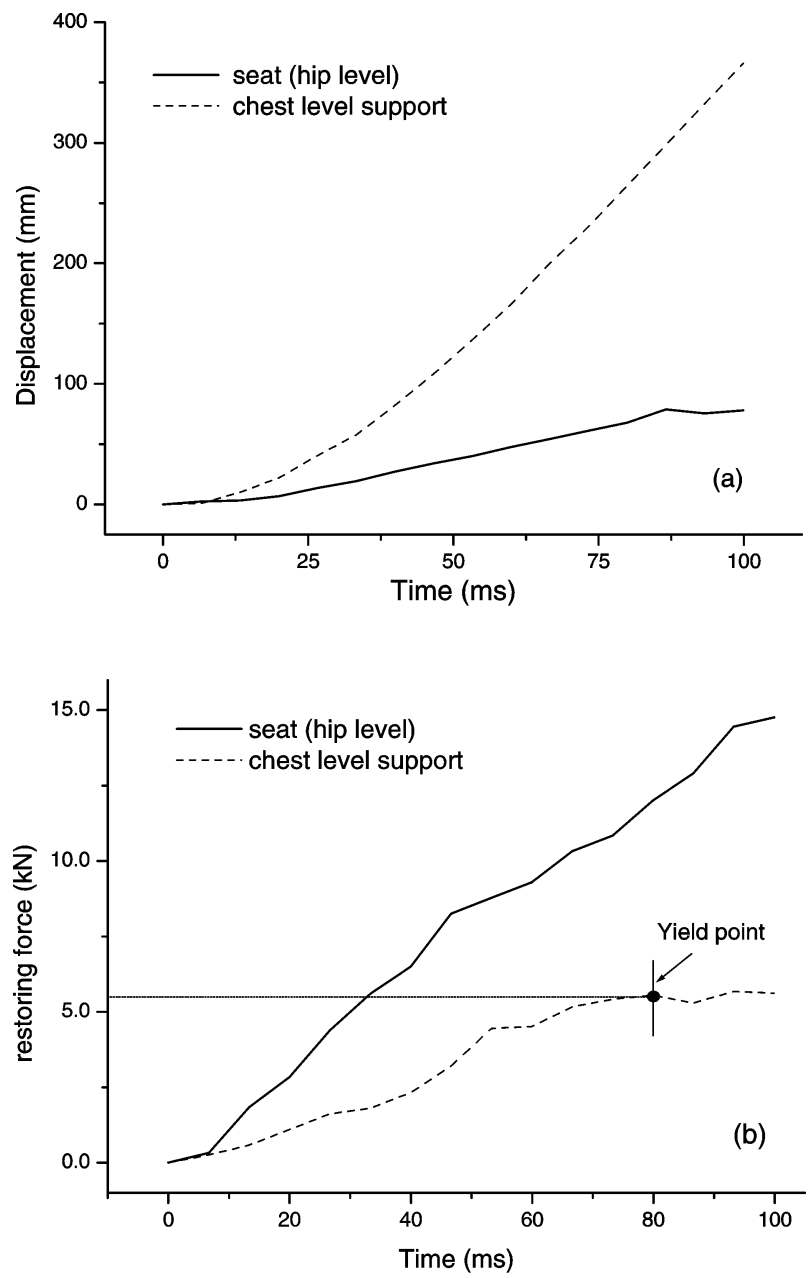

Fig. 7 PsD test: (a) horizontal displacements and (b) internal restoring forces in the seat (chest level support and hip rest) 
In contrast, a high stiffness of the seat base was detected which is important in order to ensure that the passenger could not be dangerously released during the crash.

\section{CONCLUSIONS}

The application of the PsD technique to lightweight structures in a specific automotive engineering area is presented. Given the importance of reliable dynamic analysis of some structure components in this industry concerning safety, the method has shown to be a promising research tool with low demands in terms of equipment. Moreover, the procedure provides the operator a total control of each time-step, offering the possibility of identifying initiating damage models in specific zones of the specimen, contributing to design improvement.

In fact, in order to serve the global customers, a company that produces component parts for the automobile industry (such as the production of seats), needs to subject the seats to severe real dynamic tests before its certification and consequent incorporation in major automotive brands. However, the classic test is very expensive and, in the beginning of the test it is not known if the seat fulfils the final design recommendations. Thus, the PsD method emerges as a very cheap alternative technique which can be used by companies, as an intermediate method to extensively test the seats in their own laboratories in order to obtain an optimized redesign input.

\section{ACKNOWLEDGEMENTS}

The authors would like to thank Dr Artur V. Pinto (Reaction Wall Facility of ELSA, JRC, Ispra, Italy) and Dr João G. M. Gonçalves, both working at the Joint Research Centre (JRC) of the European Communities, Ispra VA (Italy), for their technical assistance.

\section{REFERENCES}

1 Constantine, S. Finite Element Modelling in Engineering Practice, 1994 (Algor, Inc. Publishing Division, Pittsburgh, PA).

2 Pegon, P. Alternative characterization of time integration schemes. Comput. Methods Appl. Mech. Eng., 2001, 190, 2707-2727.

3 Molina, F. J., Verzeletti, G., Magonette, G., and Taucer, F. Dynamic and pseudodynamic responses in a two storey building retrofitted with rate-sensitive rubber dissipators. In Proceedings of the 12th World Conference on Earthquake Engineering, New Zealand, 2000.
4 Donea, J., Jones, P. M., Magonette, G., and Verzeletti, G. The pseudo-dynamic test method for earthquake engineering. Report EUR 12486 EN, Safety Technology Institute, Commission of the European Communities, JRC, Ispra, Establishment, Ispra, 1990.

5 Kakaliagos, A. Pseudo-dynamic testing of a full scale three-story one-bay steel moment-resisting frame. Experimental and analytical results. Report EUR 15605 EN, Safety Technology Institute, Commission of the European Communities, JRC, Ispra Establishment, Ispra, 1994.

6 Donea, J., Magonette, G., Negro, P., Pegon, P., Pinto, A.V., and Verzeletti, G. Pseudo - dynamic testing of large - scale models of civil engineering Structures at ELSA reaction Wall. In Proceedings of the 10th European Conference on Earthquake Engineering, Vienna, 1994.

7 de Melo, F. J. Q., Carneiro, J. A. O., Lopes, H. R., and Rodrigues, J. F. D. The dynamic analysis of piping systems using pseudo-dynamic techniques. J. Strain Anal., 2001, 36(5), 441-451.

8 Hilbert, H. M., Hughes, T. J. R., and Taylor, R. L. Improved numerical dissipation for time integration algorithms in structural dynamics. Earthquake Eng. Struct. Dyn., 1977, 5, 283-292.

9 Carneiro, J. O., Jalali, S., de Melo, F. Q., and Tomás, M. The effect of concrete strength and steel-barreinforcement on damping coefficient of concrete beams. In Proceedings on sixth Portuguese Conference on Seismic Engineering, Guimaraes, April, 2004, pp. 517-529 (in Portuguese).

10 Newmark, N. M. A method of computation for structural dynamics. J. Eng. Mech. Div., ASCE, 1959, 85, Ner.EM3.

11 Combescure, D. and Pegon, P. $\alpha$ - Operator Splitting time integration technique for pseudodynamic testing; Error propagation analysis. Soil Dyn. Earthquake, 1997, 16, 427-443.

12 Bathe. Finite Element Procedures, 2nd edition, 1992 (Prentice-Hall, Englewood Cliffs, New Jersey).

13 Carneiro, J. O., Melo, F. J. Q., Jalali, S., and Camanho, P. P. Analytical dynamic analysis of earthquake baseisolation structures using time history modal superposition. Proc. Instn Mech. Engrs, Part K: J. Multi-body Dynamics, 2004, 218.

\section{APPENDIX}

\section{Notation}

a acceleration vector

$\boldsymbol{a}_{i+1}$ acceleration vector determined at a discrete time instant

$B \quad$ constant

C damping factor (viscous damping)

$\boldsymbol{d}$ displacement vector

$\boldsymbol{d}_{i+1}$ displacement vector determined at a discrete time instant

$\hat{\boldsymbol{d}}_{i+1} \quad$ explicit displacement vector determined at a discrete time instant

$\boldsymbol{e} \quad$ corrective parameter 
$f_{i+1} \quad$ external force vector determined at a

$\boldsymbol{F}(t) \quad$ (as a function) external force vector

$R \quad$ radius of a traction device

$\Delta t \quad$ time step interval

$H \quad$ (length) height of seat basis and seat back

$t \quad$ time variable

holder

$i \quad$ (integer) index

$k \quad$ stiffness of a single-degree-of-freedom

k stiffness matrix (condensed)

$T \quad$ system natural period

$v \quad$ velocity vector

$v_{i+1} \quad$ velocity vector determined at a discrete

$\mathbf{k}_{i j} \quad$ matrix stiffness element

$[\mathbf{K}] \quad$ (as matrix) stiffness matrix

m lumped mass matrix (condensed)

$\mathbf{M}_{i j} \quad$ mass element (of mass matrix)

$\boldsymbol{r}$ intermediate internal restoring force vector

$\boldsymbol{r}_{i+1} \quad$ internal restoring force vector measured at a discrete time instant

$\boldsymbol{x} \quad$ intermediate displacement vector

$\beta \quad$ constant parameter

$\gamma \quad$ constant parameter

$\psi \quad$ system mode shape vector

$\omega \quad$ circular natural frequency

$\pi \quad$ constant 
ARTID......K03804

TO: CORRESPONDING AUTHOR

\section{AUTHOR QUERIES - TO BE ANSWERED BY THE AUTHOR}

The following queries have arisen during the typesetting of your manuscript. Please answer these queries by marking the required corrections at the appropriate point in the text.

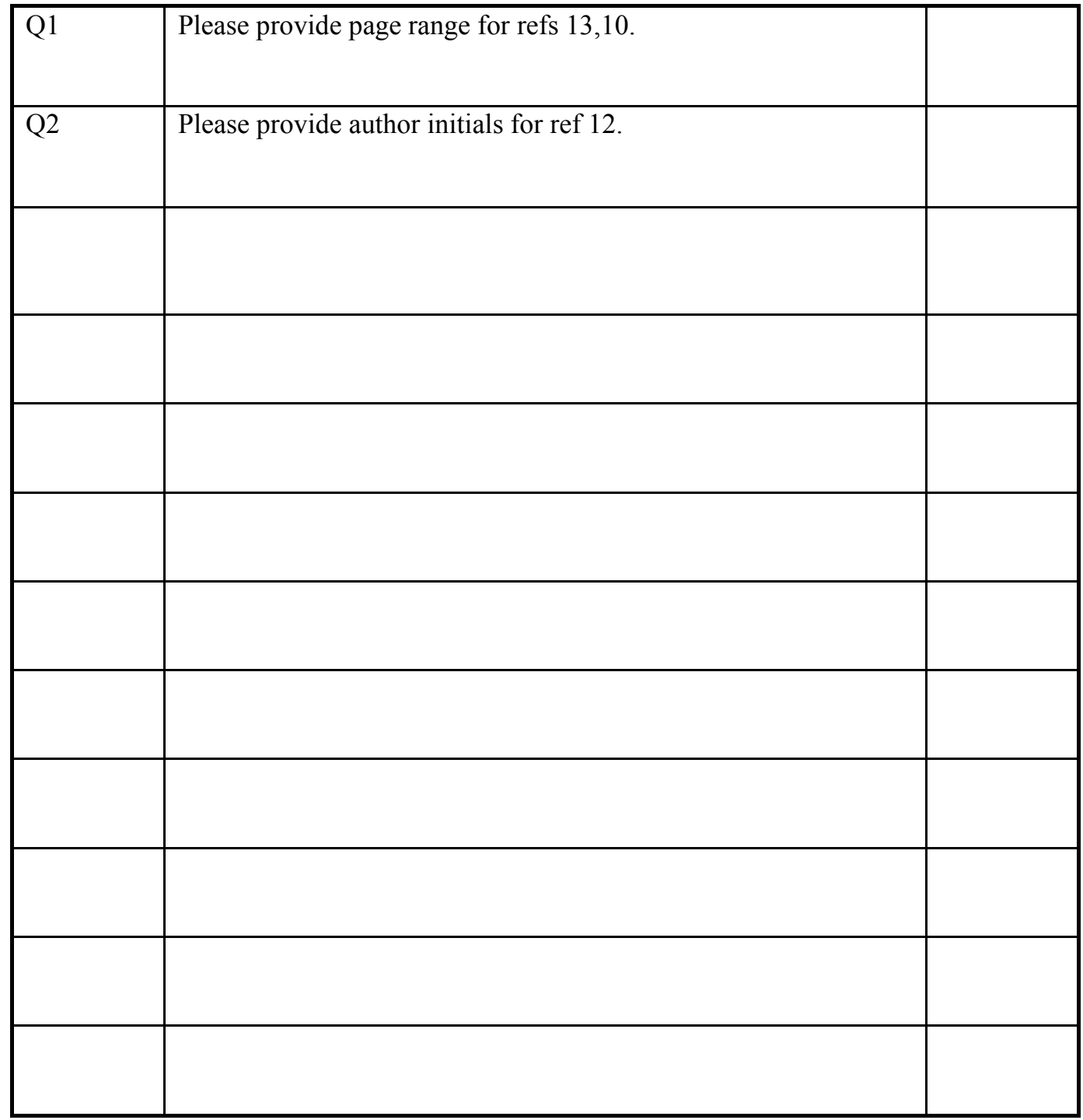


Professional Engineering Publishing Limited

1 Birdcage Walk

Westminster

London

Tel +44(0)2072227899

Fax +44 (0)20 72224557

www.pepublishing.com

\section{Journal of Multi-body Dynamics}

Proceedings of the Institution of Mechanical Engineers - Part $\mathrm{K}$

\section{Offprints/Journal Order}

To ensure that you receive the offprints or Journal you require please return this form with your corrections.

MS Number:

K03804

Number of pages: $\quad 8$

Author

Mr Joaquim Oliveira Carneiro

If you would prefer to receive a free copy of the Journal issue instead of 25 free offprints, please indicate below:

$\begin{array}{lll}\text { Quantity } & \begin{array}{l}\text { I copy of the } \\ \text { Journal } \\ \text { issue }\end{array} & \mathbf{2 5} \text { offprints } \\ \text { Cost } & \text { Free } & \text { Free }\end{array}$

Please supply offprints/1 copy of the Journal issue* and forward to the following address:

* Delete as appropriate

Mr Joaquim Oliveira Carneiro

Physics Department

Minho University

Axurem Campus

4800-058

Guimaraes

Portugal

Quotations for additional reprints will be provided on request. 